\title{
Dietary supplements containing silymarin as a supportive factor in the treatment of canine hepatopathies
}

\author{
MACIEJ GOGULSKI*,**,***, MARIE ARDOIS****, JULIA GRABSKA****, KACPER LIBERA*, \\ MAŁGORZATA SZUMACHER-STRABEL ${ }^{* * *}$, ADAM CIEŚLAK ${ }^{* * *}$, VIOLA STROMPFOVÁ ${ }^{* * *}$
}

\author{
*Department of Preclinical Sciences and Infectious Diseases, Faculty of Veterinary Medicine and Animal Science, \\ Poznań University of Life Sciences, Wołyńska 35, 60-637 Poznań, Poland \\ **University Centre for Veterinary Medicine, Faculty of Veterinary Medicine and Animal Science, \\ Poznań University of Life Sciences, Szydłowska 43, 60-656 Poznań, Poland \\ ${ }^{* * *}$ Centre of Biosciences, Slovak Academy of Sciences, Institute of Animal Physiology, \\ Šoltésovej 4-6, 040-01 Košice, Slovak Republic

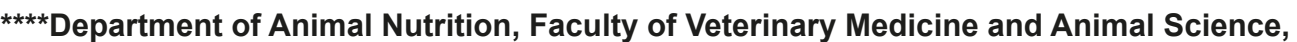 \\ Poznań University of Life Sciences, Wołyńska 33, 60-637 Poznań, Poland
}

\section{Gogulski M., Ardois M., Grabska J., Libera K., Szumacher-Strabel M., Cieślak A., Stromprová V. Dietary supplements containing silymarin as a supportive factor in the treatment of canine hepatopathies Summary}

We review canine dietary supplements, in particular silymarin and its hepatoprotective properties. Dietary supplements should be considered complements to conventional drug treatments, helping to prevent health issues, such as nutritional deficiencies and organ dysfunctions. The market for veterinary supplements is growing rapidly. Pet owners are increasingly eager to provide their animals with the best care available. The rapidly growing market of supplements is an opportunity for both the industry and veterinarians, who can introduce new treatment protocols supported by dedicated dietary supplements. The liver is one organ that can be nutritionally supported in case of dysfunction. In dogs, hepatopathies may be primary or may be secondary to an underlying problem. Toxins, drugs, and neoplasia are reported among the causes of liver dysfunctions. There are several substances with proven liver-beneficial properties. One of these is silybin, contained in silymarin, a hepatoprotector extracted from Silybum marianum. Dietary supplementation with silybin seems a reasonable tool to support a core treatment for liver disease. Laboratory tests have additionally shown that silybin supplementation helps reduce liver damage. The literature indicates that silybin acts not only as an antioxidant, but also as an antifibrotic agent and a stimulator of cell regeneration. Due to its multidirectional action, it can effectively reduce liver damage, or help prevent it. Independent of the reason for its administration (prevention or therapy), it has been reported effective in laboratory animals, dogs, and humans. This was confirmed by an observational study performed at the University Center for Veterinary Medicine at Poznań University of Life Sciences. A total of 15 dogs with diagnosed liver conditions were supplemented with silybin for 30 days. Significant improvements were reported in the liver blood markers after the supplementation period. All things considered, silybin dietary supplementation is a noteworthy strategy in prevention and treatment of liver diseases in dogs.

Keywords: dietary supplements, canine hepatopathies, silymarin, silybin

\section{Dietary supplements}

As awareness of the role of prevention in pets' health has grown among pet owners in recent decades, dietary supplements have increased in popularity (25). Such products are taken orally and contain one or more dietary ingredients intended to supplement the diet (37) and prevent nutritional deficiency (44), such as vitamins, minerals, herbs, other nutraceutical plants, amino acids, and substances such as enzymes, organ tissue, and metabolites.

Nutritional supplements not only help prevent the development of diseases, but are also a form of complementary or alternative medicine (CAM) which can be used instead of, or alongside, conventional medicinal products to reduce adverse drug effects (23). Several supplements, such as curcumin, S-adenylo-methionine 
(SAMe), silymarin, and yutan, are known to have hepatoprotective activity $(7,23,35,62)$. It should be noted, however, that some dietary supplements interact with drugs (9). Owners must therefore be sure to inform veterinarians of any medical and nutritional preparations administered to the animal, and veterinarians should have a thorough understanding of the interactions, safety, and efficacy of any such products used.

The market for dietary supplements has grown steadily for the last twenty years as a result of the increasing number of pets in households, as well as increasing knowledge among pet owners (http://www. fediaf.org/images/FEDIAF_Facts_and_Figures_2018_ ONLINE final.pdf). Botanical supplements also have the advantages of being less expensive and less toxic than conventional drugs, which may incline pet owners toward this alternative treatment (1).

In addition, nutraceuticals have become a crucial element of human health programs (24): according to Gupta et al. (24), the market for human nutraceuticals is expected to grow from 4 billion US dollars in 2019 to 10 billion US dollars by 2022 . A similarly rapid growth is likely to occur in veterinary medicine, and the global market of pet food nutraceuticals is expected to reach a value of $\$ 8.25$ billion by 2023 (https://www. mordorintelligence.com/industry-reports/pet-dietarysupplements-market).

In Europe, an estimated 80 million households own pets, $25 \%$ of which are dogs (http://www.fediaf.org/ images/FEDIAF_Facts_and_Figures_2018_ONLINE final.pdf). Furthermore, it has been reported that in Italy, $5.3 \%$ of dogs treated by veterinarians are treated with herbal supplements (1). A market analysis and forecast for the study period 2016-2020, based on the year 2019, estimated a compound annual growth rate (CAGR) of $5.6 \%$ for the dietary supplement market.

Supplements come in a range of forms, such as tablets, capsules, granules, powder, gel, gummies, liquids, and sprays https://zoobranza.com.pl/suplementy-dietypsow-i-kotow/); powders and liquids are most popular among consumers, followed closely by capsules and tablets (https://www.transparencymarketresearch.com/ pressrelease/pet-dietary-supplements-market.htm).

\section{Bioactive compounds (secondary plant metabolites)}

Currently, a large variety of supplements and pet foods supplemented with plant extracts or plant functional compounds are available. Plants contain multiple phytochemicals (bioactive compounds) with pharmacological and nutraceutical properties (55). These can be incorporated into food in small quantities and in this way administered to pets $(5,21)$. Their biological activity is associated with their ability to modulate metabolic reactions (4). They are not essential for vital functions, but it has been shown that they can have positive effects on health (5). The therapeutic potential of these molecules is based on their ability to inhibit or induce enzyme or gene expression, to inhibit or activate receptor activities, and to exert antioxidant effects by affecting energy intake. They can also reduce metabolic disorders, oxidative stress, and proinflammatory states (53). The potential health effects of bioactive compounds are highly dependent on digestive processes, which affect their bioaccessibility and bioavailability; this varies significantly between compounds (21) and thus does not always match the tissue concentration (53).

Plants produce a vast number of organic compounds, many of which do not participate directly in growth and development (28). However, they can be synthesized in response to environmental factors and serve as defense mechanisms, or play a role in specialized structures or reproductive organs $(2,61)$. Bioactive compounds are usually secondary metabolites derived from the primary metabolism of plants. Unlike primary metabolites, secondary metabolites are synthesized in small quantities (25) and, as a source of active ingredients, they are used in the production of pharmaceuticals, agrochemicals, aromatics, and food additives (2).

Phytochemicals include diverse classes of compounds with distinct chemical structures, various distributions, different ranges of concentrations in food and organisms, efficacy, specificity, and biological activity (21).

The secondary metabolites present in plants belong to four main groups: terpenes (such as quinoline, indole, purine), phenolic compounds (such as coumarins, flavonoids, lignin, tannins), glycosides (such as saponins, glucosinolates, cardiac glycosides), and alkaloids (such as hormones, carotenoid pigments, sterols, and essential oils). These present a vast diversity of properties, including antimicrobial (bactericidal and bacteriostatic), antihelmintic (tannins and saponins), antioxidant, anti-tumorous, anti-inflammatory, immunostimulant (phenolic compounds, alkaloid saponins, and terpenes), and detoxification-supporting properties. They can also take part in physiological functions, such as the transport of toxic nitrogen and protection against UV rays, as well as enhancing immune responses (27).

From the nutritional and physiological point of view, special attention should be drawn to antioxidants, which support the natural defense mechanisms of cells and are widely considered as preventive against numerous diseases (including hepatopathies). Excessive oxidation may also cause adverse changes in the quality factors of food and may contribute to toxic effects (61).

The presence or absence of these properties depends on factors such as the kind of plant, the type of bioactive compound, the presence of other secondary metabolites, the quantity consumed, and the ingestion frequency (27).

Although phytogenic substances are of natural origin and therefore perceived as safe, it should not be 
forgotten that they may also be toxic, particularly to the liver and other excretory organs (61). There are no precise data on the recommended number or dosage of intakes, or the effectiveness and safety of particular bioactive compounds, but it has been shown that, as part of a balanced diet, they may produce beneficial health effects (5).

\section{Silybin: characteristics and mechanism of action}

One secondary plant metabolite that exhibits health-beneficial properties is silybin. Silybin, with its isomers silybin A and B isomers, is one of the most active flavonolignans present in the extract from milk thistle, Silybum marianum. Along with other flavonolignans (isosilybin, silidianin and silicristin), it forms a complex known as silymarin. It is present in every structure of the plant and is mostly concentrated in the fruit. Standard extract are $60 \%$ to $80 \%$ silymarin (12).

Silybin displays several hepatoprotective actions, such as an antioxidant effect and an ability to impair hepatic fibrogenesis, while accelerating liver regeneration by contributing to the epigenetic regulations. Moreover, it increases the bile output of hepatocytes, which produce ursodeoxycholic acid, a bile acid known for its hepatoprotective potential (12).

By interfering with the formation of oxidant toxins, accelerating their degradation or disposal, and preventing toxins from connecting to their receptors or binding sites, silymarin inhibits lipid peroxidation and the synthesis of reactive oxygen species (ROS). ROS play a key role in the process of inflammation, as their presence results in cytokine liberation and the activation of tissue macrophages (32). Moreover, it has been found that silymarin also interacts with cell and mitochondrial membranes, modifying the flux of substances through them $(12,14)$.

In liver injury, tissue macrophages called Kupffer cells are activated. Their mitochondria produce large amounts of ROS as a result of lipid peroxidation (12). Malondialdehyde (MDA) and mitochondrial desferrioxamine (DFO) can be used as indicators of lipid peroxidation (47). It has been found after treatment of liver hemochromatosis with silybin that the MDA concentration in the mitochondria of these cells was reduced to negligible levels, while DFO levels remained almost unchanged, compared to the control group. Moreover, it has been found that silybin treatment prevents membrane potential disturbance and maintains an appropriate level of activity of the cell respiration cycle and of its cytochrome $\mathrm{c}$, which in turn allows the levels of ATP, an indicator of cell viability, to remain steady (47). Through its mode of action, silybin prevents mitochondrial dysfunction, thus maintaining the proper functioning of the cell and its membrane.

Liver intoxication by peroxidation inducers, such as paracetamol, ethanol, or phenylhydrazine, results in an accelerated cell respiratory rate and the release of toxic thiobarbituric acid reactive substances (TBARS) (20). It has been investigated that reduced glutathione (GSH) pool required, in response to the provoked oxidative stress, is not sufficient, consequently depletion occurs. However, model rats injected intravenously (IV) with silybin at a dose of $50 \mathrm{mg} / \mathrm{kg}$ showed an important diminution in oxygen consumption rate, along with stabilized GSH levels in the cells (20). It was thus demonstrated that silybin has several effects, not only on the concentration of free radicals, but also on processes in which both glutathione and superoxide dismutase are involved. Moreover, it inhibits lipoxygenase, thus limiting lipid peroxidation (20).

It is suspected that silymarin administered at high doses, may also interfere in the phase I of detoxification pathway, by inhibiting the function of cytochrome p450 (CYP450), preventing the formation of oxidant toxins $(12,20)$. It is worth noting that these features make silybin a potential competitor for drugs which bind to CYP450.

However, as has been shown by Ying Xie et al., silybin has been shown in multiple studies to have little or no effect on drug metabolism and sometimes, even improving the availability of active substances (67). For example, it has been shown to have no influence in humans taking a combination of aminopyrine and phenylbutazone or midazolam. A moderate effect was observed in the case of metronidazole, with a reduction of about $30 \%$ of the area under curve (AUC) $(66,67)$ as well as a reduced half-life and increased clearance (66) and - in the case of indinavir - with a reduction of $9 \%$ of AUC (66). On the other hand, silymarin has been shown to have a significant influence on talinolol, which increased its AUC by $36 \%$, and on domperidon, whose AUC increased fivefold in the presence of silymarin. Furthermore, silybin has been shown to be a highly selective inhibitor of uridine diphosphoglucuronyl transferase 1A(UGT1A) (66), which catalyzes glucuronidation during phase II drug metabolism. Hence, the distribution ratios of pyrazinoic acid, an active metabolite of pyrazinamide, have been found to be affected by silybin, which had negative effects on its elimination (66). In addition, Jhy-Wen Wu et al. found that the reduction of the P-glycoprotein (P-gp) present in Caco-2 intestinal epithelial cells was inhibited by silymarin, which increased the intestinal absorption of drugs otherwise excreted back into the intestine lumen (66). For example, the bioavailability of digoxin, a cardiac glycoside, was increased after the administration of $50 \mu \mathrm{M}$ silymarin in vitro, but the correlation was not demonstrated in vivo.

However, the properties of silybin mean that treatment with it may result in a diminished oxidative cell damage, indirectly preventing repairing processes, such as fibrosis.

The transcription factor nuclear factor kappa B (NF$\kappa \beta)$ is a protein complex inducing the translation of ge- 
netic material and the transcription of survival genes in response to inflammatory factors (48). In hepatocytes, NF- $\kappa \beta$ can be activated by viral or bacterial products, proinflammatory cytokines, oxidants, and ROS. Once activated, it binds to the promoter region of DNA which codes for the protein of the inflammatory response. On one hand, there is evidence that silymarin inhibits this process through two mechanisms (48). Firstly, it may inhibit the phosphorylation of NF- $\kappa \beta$, keeping it in an inactive state. Secondly, it may also prevent the nuclear translocation of the active subunits of NF- $\kappa \beta$ (p50 and p65). The exact mechanisms of these processes are still unknown. On the other hand, a study by Au et al. revealed that a combination of S-adenosylmethionine and silybin exerts anti-inflammatory and antioxidant effects through two separate signaling pathways namely, by reducing the cytokine-induced production of PGE2, IL-8 and MCP-1, and subsequently inhibiting NF-BK (6), which is accompanied by an increase in antioxidant enzyme-reduced glutathione (GSH). Nonetheless, it should be underlined that silymarin does not inhibit the binding of NF- $\kappa \beta$ to DNA. This mechanism also indirectly prevents liver fibrosis, as it results in the survival of liver cells (48).

Still, the direct antifibrotic properties of silybin are due to the fact that it not only inhibits the activation, differentiation, and mitosis of extracellular compoundsynthesizing cells in the liver (stellate cells), but it also interferes with the type 1 collagen synthesis pathway and decreases the production of metalloproteinase-1 tissue inhibitor (TIMP1), both of which are expressed by activated stellate cells $(10,39)$. The decrease in TIMP1 allows an increase in matrix metalloproteinase-1 (MMP1), a collagenase known for dissolving extracellular matrix (ECM), and particularly collagen type 1 , to allow tissue remodeling (10). It has been demonstrated that a treatment of silybin administered at $50 \mathrm{mg} / \mathrm{kg} /$ day for 6 weeks decreased the amount of collagen accumulated in an inflamed liver by $12 \%$ and stimulated the excretion of bile containing ursodeoxycholic acid (12).

Silybin stimulates cell regeneration by acting directly on the process of DNA transcription. It has been demonstrated in vivo on rats that a dose of $10 \mathrm{mg} / \mathrm{kg}$ of silybin increased the amount of proteins synthesized by $40 \%(60)$. The result indicates that the effect of silybin was greater at the higher dose of $25 \mathrm{mg} / \mathrm{kg}$. The translation on which silybin has an impact results from the intensified synthesis of ribosomal RNA (rRNA) due to the stimulation of polymerase I. Moreover, Sonnenbichler and Zelt (60) found an increase in the transcription of $45 \mathrm{~S} \mathrm{RNA}$, which is a precursor for the further synthesis of $5.8 \mathrm{~S}, 18 \mathrm{~S}$, and $28 \mathrm{~S}$ RNA, which are eukaryotic ribosome constituents. As a result, more ribosomes are active in the nucleus, which increases the translation potential and thereby the protein content of the cell.
In addition, the results of Sonnenbichler and Zelt (60) indicate that the DNA replication process is also stimulated by silybin in hepatectomized rats. Using radioactive thymine, they found that, along with the stimulation of protein synthesis, silybin treatment also enhanced DNA replication. Doses of $27 \mathrm{mg} / \mathrm{kg}$ increased DNA synthesis by $25-30 \%$.

As the synthesis of both proteins and DNA is increased, cells mature more quickly and progress more quickly to the next stage of the cell cycle. The stimulation of hepatocyte replication certainly favors recovery from liver injury (60).

It is worth noting that silymarin can exhibit a synergistic effect when combined with other plant materials, such as garlic (56) or Phyllanthus amarus extract (68). Interestingly, administration of amiodarone (an antiarrhythmic drug with toxicity due to the generation of free radicals) in combination with silymarin has been shown to potentiate its antiarrhythmic action in induced atrial flutter. However, the mechanism of this action still needs to be clarified (63).

The pharmacological characteristics of silymarin should also be kept in mind when using this treatment. Silymarin can be administered either per os (PO) or $\mathrm{IV}$, and is not soluble in water (22). It is thus mostly excreted with the bile $(80 \%$ and $40 \%-45 \%$ during the first 48 hours after IV and PO administration, respectively), and only trace amounts are found in urine $(8 \%$ and 2\%-5\% during the first 48 hours after IV and PO administration, respectively). The results of Persiani et al. indicate that silybin hemisuccinate is strongly bound to plasma proteins, and the extent of binding is similar for different animal species, including dogs, rats, mice, and humans. Thus, no corrections for an unbound drug fraction are needed when animals are involved in toxicological studies for human medicine (46). It is worth noting that silybin in urine is found unmodified, whereas only metabolites are excreted with bile in rats (13), regardless of the mode of administration (20). Moreover, the authors show that there is a linear relation between the quantities administered and excreted (dose: $\leq 20 \mathrm{mg} / \mathrm{kg}$ ).

PO bioavailability is low, which is shown by its excretion with the bile accounting for only 2 to $3 \%$ of the total oral dose (20). It has been found that the bioavailability of silymarin increases when administered orally in the form of liposome suspensions. Yan-yu et al. carried out a study on beagle dogs to examine the possibility of increasing the gastrointestinal absorption of silymarin encapsulated with proliposome (70). The study revealed that the oral administration of silymarin proliposome could lead to a higher bioavailability than the administration of pure silymarin or silybin (70). Pharmacokinetic studies conducted with dogs by Filburn et al. revealed that the bioavailability of silybin administered with a phosphatidylcholine complex (phytosome) is higher than that of the conventional, 
nonphytosome form (19). Similar studies carried out on humans and rats demonstrated the greatly improved bioavailability of silybin combined with phosphatidylcholine, due to better transition from a hydrophilic environment to a lipophilic environment of the cells $(31,36)$.

The plasma peak occurs 4 to 6 hours after administration, with an elimination half-life $(\mathrm{T} 1 / 2)$ of 6 to 8 hours (13). Peak excretion in urine takes place 1 hour after PO administration (20).

Following administration, most components of silymarin are conjugated in the liver with sulfate and glucuronic acid $(13,20)$. They reach the bloodstream before being excreted with the bile as conjugates and undergoing enterohepatic recirculation (20). From the intestinal lumen, $35 \%$ of the conjugate undergoes reuptake, and peak plasma concentration is reached 30 minutes after ingestion.

Silybin is found distributed among several tissues. Half an hour following administration, it is found mostly in the organs of the digestive tract, such as the liver, the pancreas, and the stomach (20), which have significant glandular activity, but is also found in the lungs. After 30 minutes, concentrations of $8.8( \pm 1.6)$ $\mu \mathrm{g} / \mathrm{g}$ in the liver, $123( \pm 21) \mu \mathrm{g} / \mathrm{g}$ in the stomach, and $5.8( \pm 1.1) \mu \mathrm{g} / \mathrm{g}$ in the pancreas $(20)$ are found. These data suggest that the highest concentration is found in the stomach, but there seem to have been no studies on its role and mode of action, or the reason for its presence here.

Silymarin appears to be a safe nutraceutical, as its $50 \%$ lethal dose $\left(\mathrm{LD}_{50}\right)$ for dogs is $140 \mathrm{mg} / \mathrm{kg}$ IV (which increases as the rate of infusion decreases) and $10 \mathrm{~g} / \mathrm{kg} \mathrm{PO}$, with a maximum tolerated dose of $300 \mathrm{mg} /$ $\mathrm{kg}$ (20). If LD50 is exceeded, the cause of death appears to be cardiovascular. Moreover, silymarin seems to have no effect on embryonic development. Hence, we can conclude that the acute, subacute, and chronic toxicities of silymarin are very low (20). Silymarin has been reported as a potent inhibitor of the thyroid hormone transporter MCT8, which suggests it may not be safe as an adjuvant therapeutic in liver diseases (29), but it is generally well-tolerated by both animals and humans; and the most prevalent adverse reactions to silymarin are diarrhea, abdominal pain, pruritus, and nausea (59).

It is important to bear in mind, however, that these data were collected for humans, and, to the authors' knowledge, there is no data available on the herbaldrug interaction of silymarin in dogs.

Some animal data are available for rats and rabbits that were given silymarin as a pretreatment; these show that it resulted in an increase of the AUC and maximum plasma concentration $\left(\mathrm{C}_{\max }\right)$ for nitrendipine, and in elevated exposure of methadone (67).

Information found on the Internet suggests that the following preparations containing silybin are commer- cially available in Poland: Hepaxan (Vebiot, Warsaw, Poland), Hepatoforce (Vetfood, Łódź, Poland), BARFeed Milk Thistle (Vetfood, Łódź, Poland), BARF Silybum Marianum (Canvit, Chrášt’any, Czech Republic), Silimarina (DogShield, Skierniewice, Poland), HepaDol (Dolfos, Piotrków Trybunalski, Poland), Hepachol S (Eurowet, Kościelna Wieś, Poland), Slimvet (Eurowet, Kościelna Wieś, Poland), Hepatikrill Cane (iloVet, Dąbrówka, Poland), Silivet (Biofaktor, Skierniewice, Poland) and RawDietLine Ostropest (Pokusa, Wieliczka, Poland). The wealth of commercially available preparations suggests a significant demand for this type of product on the Polish veterinary market. We believe that this market, which includes silybin preparations, will grow rapidly, in line with global trends.

\section{Hepatopathies}

Both infectious and noninfectious factors should be taken into account in canine liver diseases. The infectious etiologic agents reported in the literature are limited to Canine adenovirus-1 (CAV1), a known cause of acute hepatitis, also known as infectious canine hepatitis (ICH), and Rubarth's Disease (18), and Leptospira spp. (8), and leishmaniasis (50). However, noninfectious agents are believed to be the predominant cause of hepatopathies in dogs: factors such as toxins, certain drugs, and neoplasia (26) have been described (8). Hepatotoxin is a general term for a substance that may damage the liver. For example, drugs such as diethylcarbamazine/oxibendazole, phenobarbital, and trimethoprim/sulfadiazine may be hepatotoxic (11). Some causes of hepatopathies are congenital, such as defects in copper metabolism leading to a chronic form of hepatitis that has been described in several breeds of dogs (15). Copper storage disease was first described in the Bedlington Terrier and then in the Dalmatian, Doberman Pinscher, and Labrador Retriever (15). Another type of inherited liver malformation is vascular abnormalities in the form of portosystemic shunts (54). Agenesis of a hepatic lobe is an extremely rare congenital anomaly, and only one case has been reported in the veterinary literature in a dog (41). However, the causes of most hepatitis cases are unknown and are considered idiopathic (18).

Hepatopathies can be further classified. Generally, hepatopathy can be a primary problem or may be secondary to other conditions, which should be dealt with first. For example, very pale mucous membranes in the presence of icterus indicate that the liver dysfunction is secondary to hemolytic anemia. Furthermore, regularly encountered forms of primary hepatopathy in dogs include acute hepatitis and chronic hepatitis (with or without cirrhosis). Less frequently encountered forms are lobular dissecting hepatitis, granulomatous hepatitis, and eosinophilic hepatitis (18). It is important to note that WSAVA has developed diagnostic guidelines 
regarding liver diseases. For example, these define acute hepatitis morphologically as a combination of inflammation, hepatocellular apoptosis, and necrosis, and, in some instances, regeneration (52).

In addition to dysfunctions of liver parenchyma, the biliary system can also be affected, with conditions such as cholecystitis, gallbladder mucocele, and gallbladder neoplasia having been reported in dogs (40).

\section{Markers of liver disorders}

According to the World Health Organization, a biomarker is "a chemical, its metabolite, or the product of an interaction between a chemical and some target molecule or cell that is measured in the human body" (http://www.who.int/ceh/capacity/biomarkers. pdf?ua $=1)$. When tissue cells are damaged or broken down, biomarkers are released into the circulation.

Novel methods of biochemical analysis now allow the discovery and measurement of a large array of biomarkers, not only in urine, saliva, tissues, or feces, but also in blood.

Regular monitoring of the levels of biomarkers in blood is crucial for the early diagnosis of pathological conditions. Nonetheless, for a complete diagnosis, a competent practitioner needs to interpret blood work results, as several markers - depending on their concentrations and the ratio between them - may lead to different conclusions.

Tab. 1. Summary of the most significant liver markers in dogs

\begin{tabular}{|l|l|l|l|}
\hline \multicolumn{1}{|c|}{ Liver markers } & \multicolumn{1}{|c|}{ Type } & Abbreviation & \multicolumn{1}{|c|}{ Reference } \\
\hline Alanine transferase & enzymatic & ALT & eClinpath, 2013' \\
\hline Aspartate transaminase & enzymatic & AST & Ozer, 2008 (43) \\
\hline Arginase & enzymatic & ARG & Ozer, 2008 (43) \\
\hline Alkaline phosphatase & enzymatic & ALP & Bexfield, 2016 (8) \\
\hline Butyrylcholinesterase & enzymatic & BChe & Carmo, 2015 (16) \\
\hline Gamma-glutamyltransferase & enzymatic & GGT & Ozer, 2008 (43) \\
\hline Glutamate dehydrogenase & enzymatic & GLDH & eClinpath, 20132 \\
\hline Malate dehydrogenase & enzymatic & MDH & Ozer, 2008 (43) \\
\hline Sorbitol dehydrogenase & enzymatic & SDH & Gerlach, 1974 (22) \\
\hline Albumin & protein & ALB & eClinpath, 2013 \\
\hline Alpha-fetoprotein & protein & AFP & Yamada, 1994 (69) \\
\hline Fibrinogen & protein & FIB & Redman, 2001 (51) \\
\hline Ammonia & metabolites & NH & Aldridge, 2015 (3) \\
\hline Hyaluronic acid & metabolites & HA & Kanemoto, 2009 (30) \\
\hline Total bilirubin & metabolites & TBIL & Ozer, 2008 (43) \\
\hline Blood urea nitrogen & others & BUN & IDEXX, 20134 \\
\hline Extracellular vesicles & others & EVs & Povero, 2014 (49) \\
\hline MicroRNA & others & - & Koenig, 2016 (33) \\
\hline
\end{tabular}

Explanations: ${ }^{1} \mathrm{http}: / / \mathrm{www}$. eclinpath.com/chemistry/liver/liver-injury/ alanine-aminotransferase, ${ }^{2} \mathrm{http}: / / \mathrm{www}$. eclinpath.com/chemistry/liver/ liver-injury/glutamate-dehydrogenase, ${ }^{3} \mathrm{http}: / / \mathrm{www} . e c l i n p a t h . c o m /$ chemistry/proteins/albumin, ${ }^{4}$ http://www.idexx.eu/globalassets/documents/ parameters/8071-us-bun-interpretive-summary.pdf.
The most specific markers of liver injury may be of either enzymatic or nonenzymatic origin, the latter including proteins, metabolites, and other circulating compounds (Tab. 1).

Those used on a daily basis in veterinary practice are alanine transferase (ALT), aspartate transaminase (AST), alkaline phosphatase (ALP), bilirubin and albumin (42) and, to a smaller extent, gamma-glutamyltransferase (GGT), sorbitol dehydrogenase (SDH), and glutamate dehydrogenase (GLDH) (13).

\section{Is silybin supplementation always effective?}

In the veterinary literature, there is limited information on the hepatoprotective properties of silybin in dogs. A few older studies investigated the potential of silybin in protecting the liver against the experimental toxicity of Amanita phalloides mushrooms (64) or tetrachloromethane-induced liver disease (45). The results were not consistent. Vogel et al. (64) observed an increased survival rate and fewer clinical signs of poisoning, whereas according to Paulova (45) the effectiveness of silybin in protecting the liver was low. In more recent studies, Kocaturk et al. observed a significant reduction in the activity of hepatic enzymes, including ALT and ALP, in dogs supplemented with a combination of SAMe and silybin in the course of endotoxemia experimentally induced with LPS (34). This is consistent with a case report by other researchers who also observed reduced values of hepatic markers, such as ALT, AST, ALP, and LDH, in a bitch with stanozol-induced hepatotoxicity treated with silymarin (38). The authors of another study examined the effect of including several plant-based supplements in the diet of 74 dogs. They found that, with a daily silymarin dose of $10 \mathrm{mg}$ per $\mathrm{kg}$ of live weight, dogs with increased ALT and GTP activities on the first day of the experiment showed a decrease in these parameters sixty days later (55). These researchers also reported an increase in both plasma paraoxonase (PON) and the mitochondrial gene coding for mitochondrial superoxide dismutase 2 (SOD2), which supported the thesis about the antioxidant activity of silymarin (55). Another study (58) suggests that a combination of S-adenosylmethionine and silybin can minimize increased liver enzyme activity during chemotherapy in cancer treatment in dogs.

This is in accordance with our observational study in which we also noted a significant reduction in liver enzyme activity in dogs with liver dysfunctions. Between April 2018 and April 2019, fifteen dogs were selected for analysis from dogs diagnosed with liver disease at the University Center for Veterinary Medicine at the Poznań University of Life Sciences. Seven male and eight female dogs of different breeds 
with a mean age of $10 \pm 1.2$ years and a mean body weight of $20 \pm 2 \mathrm{~kg}$ were used. The diagnosis was based on clinical examination and elevated values of liver enzymes. The dog owners were advised to begin supplementing their pets' diet with a herbal preparation containing silybin as a bioactive compound (Hepaxan, Vebiot, Poland) at a dose of $28.3 \mathrm{mg}$ silybin per $10 \mathrm{~kg}$ BW (as recommended by the manufacturer). Blood samples for determining a liver panel were collected on the first day of supplementation and thirty days later. Blood parameters (ALBUMIN, ALT, AST, TBIL, GLDH, GGT, ALP, BUN/ UREA, $\alpha A M Y L A S E$, LDH, UREA, and GLOBULIN) were determined using a commercially available highthroughput immunoassay (IDEXX SDMA Test; IDEXX Laboratories, One IDEXX Drive, Westbrook, Maine 04092, USA). The results (mean value, standard error of means, and P-value) are given in Table 2. All data were analyzed using the SAS statistical software (Univ. Edition, version 9.4). The results were tested with an independent $t$-test in which the means of both groups (at days 1 and 30 of supplementation) were compared using the PROC TTEST procedure. The means were considered significantly different at $\mathrm{P}<0.05$. All values are shown as means with pooled standard errors of means.

The results clearly demonstrate that silybin supplementation positively affected the condition of the liver (Tab. 2). The values of the enzymatic liver markers that were analyzed - including AST, GLDH, GGT, and ALP - decreased significantly and were within physiological norms. ALT values were high before supplementation and, despite decreasing, remained above the norm. Furthermore, a slight increase in albumin and globulin concentrations was observed. On the other hand, the concentration of UREA decreased. The supplementation did not influence the values of LDH, $\alpha$ AMYLASE, or BUN/UREA. Generally, silybin supplementation seems to be a reasonable strategy in dogs with liver disease but, in some cases, depending on diagnosis, the time of administration should be extended.

A hepatoprotective effect of silymarin was observed in other animal species in an experiment by Eminzade et al., who administered it concurrently with drugs used in the chemotherapy of tuberculosis in rats (17). Intragastric administration of silymarin with antituberculosis drugs in two groups of rats significantly decreased serum ALT, AST, and ALP activities (17). Another experiment suggested a protective effect of silymarin in rats treated with cisplatin, a highly hepatoxic and nephrotoxic drug used in the treatment of cancer (57). After administration of cisplatin, an increase in hepatic enzymes, such as ALT, AST, and ALP, was seen in the treatment group. However, a group given both cisplatin and silymarin showed a significant decrease in these parameters (57).

In human medicine, silybin demonstrates therapeutic potential in cases of acute poisoning due to Amanita phalloides (death cap mushroom), alcoholic cirrhosis, and hepatopathy from exposure to toxic levels of toluene or xylene (65). However, it is considered ineffective against viral hepatitis (65).

Generally, silybin administration should be considered as a supportive therapy in liver diseases. Our research clearly indicates that silybin at a dose $28.3 \mathrm{mg}$ silybin per $10 \mathrm{~kg} \mathrm{BW}$ may be effective in improving enzymatic liver markers. It should be underlined, however, that only a thorough diagnostic process can identify the cause of liver dysfunction; concurrent diseases must also be taken into account. When liver dysfunction is secondary to an underlying primary condition, the primary condition should be managed 
first with causal treatment, while silybin supplementation may lessen clinical signs or reduce liver enzyme activities, improving the patient's general condition.

\section{References}

1.Abdelrahman N., El-Banna R., Arafa M. M., Hady M. M.: Hypoglycemic efficacy of Rosmarinus officinalis and/or Ocimum basilicum leaves powder as a promising clinico-nutritional management tool for diabetes mellitus in Rottweiler dogs. Vet. World 2020, 13, 73.

2. Ákos M.: Medicinal and Aromatic Plants of the World (eBook). Springer 2014.

3. Aldridge D. R., Tranah E. J., Shawcross D. L.: Pathogenesis of hepatic encephalopathy: Role of ammonia and systemic inflammation. J. Clin. Exp. Hepatol. 2015, 5, 7-20.

4. Angiolillo L., Del Nobile M. A., Conte A.: The extraction of bioactive compounds from food residues using microwaves. Curr. Opin. Food Sci. 2015, 5, 93-98.

5. Astley S., Finglas P.: Nutrition and Health. Reference Module in Food Science. Elsevier, Amsterdam 2016

6. Au A. Y., Hasenwinkel J. M., Frondoza C. G.: Hepatoprotective effects of S-adenosylmethionine and silybin on canine hepatocytes in vitro. J. Anim. Physiol. An. N. 2013, 97, 331-341.

7. Bahmani M., Shirzad H., Rafieian S., Rafieian-Kopaei M.: Silybum marianum: Beyond Hepatoprotection. J. Evid.-Based Complementary Altern. Med. 2015, 20, 292-301.

8. Bexfield N.: Canine idiopathic chronic hepatitis. Vet. Clin. North Am. Small Anim. Pract. 2017, 47, 645-663.

9. Boothe D. M.: Small Animal Clinical Pharmacology \& Therapeutics (eBook), Second Edition. Elsevier Health Sciences 2011.

10. Brinckerhoff C. E., Matrisian L. M.: Matrix metalloproteinases: A tail of a frog that became a prince. Mol. Cell Biol. 2002, 3, 207-214.

11. Bunch S. E.: Hepatotoxicity associated with pharmacologic agents in dogs and cats. Vet. Clin. North Am. Small Anim. Pract. 1993, 23, 659-670.

12. Center S. A.: Metabolic, antioxidant, nutraceutical, probiotic, and herbal therapies relating to the management of hepatobiliary disorders. Vet. Clin. Small Anim. 2004, 34, 67-172.

13. Corchete P.: Silybum marianum (L.) Gaertn: The source of silymarin. Bioact Mol. Med Plant. 2008, 123-148.

14. Cuendet M. J., Pezzuto M.: The role of cyclooxygenase and lipoxygenase in cancer chemoprevention. Drug Metabol. Drug Interact. 2000, 109-157.

15.Dirksen K., Fieten H.: Canine copper-associated hepatitis. Vet. Clin. North Am. Small Anim. Pract. 2017, 47, 631-644.

16. Do Carmo G. M., Crivellenti L.Z., Bottari N.B., Machado G., Borin-CrivellentiS. Moresco R. N., Duarte T., Duarte M., Tinucci-Costa M., Morsch V. M., Schetinger M. R., Stefani L. M., Da Silva A. S.: Butyrylcholinesterase as a marker of inflammation and liver injury in the acute and subclinical phases of canine ehrlichiosis. Comp. Immunol. Microbiol. Infect. Dis. 2015, 15, 16-21.

17. Eminzade S., Uraz F., Izzettin F. V.: Silymarin protects liver against toxic effects of anti-tuberculosis drugs in experimental animals. Nutr. Metab. (Lond.) 2008, 5, 18

18. Favier R. P.: Idiopathic hepatitis and cirrhosis in dogs. Vet. Clin. North Am. Small Anim. Pract. 2009, 39, 481-488.

19. Filburn C. R., Kettenacker R., Griffin D. W.: Bioavailability of a silybinphosphatidylcholine complex in dogs. J. Vet. Pharmacol. Therap. 2007, 30 132-138.

20. Fraschini F., Demartini G., Esposti D.: Pharmacology of silymarin. Clin. Drug Invest. 2002, 1, 51-65.

21. Galanakis C. M.: Introduction in Nutraceutical and Functional Food Components. Academic Press 2017, p. 1-14.

22. Gerlach U., Hiby W: Sorbitol Dehydrogenase: Methods of Enzymatic Analysis (Second Edition). Academic Press 1974, p. 569-573.

23. Glynn J., Bhikha R.: Herbal products and conventional drugs: An uneasy alliance. Bangladesh J. Med. Sci. 2018, 4, 193-198.

24. Gupta R. C., Srivastava A., Lall R. (ed.): Nutraceuticals in Veterinary Medicine. Springer 2019

25.Habior A.: Zioła i suplementy diety a ryzyko uszkodzenia wątroby. Gastroenterologia Kliniczna 2012, 4, 59-68.

26. Hammer A. S., Sikkema D. A.: Hepatic neoplasia in the dog and cat. Vet. Clin. North Am. Small Anim. Pract. 1995, 25, 419-435.

27. Hernandez-Alvadaro J., Zaragoza-Bastida A., Lopez-Rodriguez G., PelaezAcero A., Olmedo-Juarez A., Rivero-Perez N.: Antibacterial and antihelmintic activity of plant secondary metabolites: Approach in veterinary medicine. Abanico Veterinario 2018, 8, 14-27.

28. Jimenez-Garcia S. N., Vazquez-Cruz M. A., Guevara-Gonzalez R. G., TorresPacheco I., Cruz-Hernandez A., Feregrino-Perez A. A.: Current approaches for enhanced expression of secondary metabolites as bioactive compounds in plants for agronomic and human health purposes: A review. Pol. J. Food Nutr. Sci. 2013, 63, 67-78.

29. Johannes J., Jayarama-Naidu R., Meyer F., Wirth E. K., Schweizer U., Schomburg L., Köhrle J., Renko K.: Silychristin, a flavonolignan derived from the milk thistle, is a potent inhibitor of the thyroid hormone transporter MCT8. Endocrinology 2016, 157, 1694-1701

30. Kanemoto H., Ohno K., Sakai M., Nakashima K., Takahashi M., Fujino Y., Tsujimoto $H$.: Blood hyaluronic acid as a marker for canine cirrhosis. J. V. Med. Sci. 2009, 17.

31. Kidd P., Head K.: A Review of the bioavailability and clinical efficacy of milk thistle phytosome: A silybin-phosphatidylcholine complex (siliphos). Altern. Med. Rev. 2005, 10, 193-203.

32. Kobylarek D., Iwanowski P., Lewandowska Z., Limphaibool N., Szafranek S., Labrzycka A., Kozubski W.: Advances in the potential biomarkers of epilepsy. Front. Neurol. 2019, 10, 685 .

33. Koenig E. M., Fisher C., Bernard H., Wolenski F. S., Gerrein J., Carsillo M., Gallacher M., Tse A., Peters R., Smith A., Meehan A., Tirrell S., Kirby P.: The Beagle dog MicroRNA tissue atlas: Identifying translatable biomarkers of organ toxicity. BMC Genomics 2016, 1-13.

34. Kotacurk M., Eralpinan O., Levent P., Yilmaz Z.: Protective effect of S-adenosylmethionine (SAMe) and silybin on hepatorenal and hemostatic function in dogs with endotoxemia. Turk. J. Vet. Anim. Sci. 2016, 40, 788-796.

35. Lalhriatpuii T: : HPTLC fingerprint in herbal drug formulations in Herbal Medicine in India. Springer, Singapore 2020, 337-362.

36. Loguercio C., Festi D.: Silybin and the liver: From basic research to clinical practice. World J. Gastroenterol. 2011, 17, 288-2301.

37. Messonnier S.: Nutritional Supplements of the Veterinary Practice: A Pocket Guide, Electronic Edition. American Animal Hospital Association Press 2012, p. 9-13.

38. Mosallanejad B., Avizeh R., Varzi H. N.: Successful treatment of stanozolol induced-hepatotoxicity with silymarin in a bitch. Asian J. Anim. Sci. 2011, 5, 213-218.

39. Murphy F. R., Issa R., Zhou X., Ratnarajah S., Nagase H., Arthur M. J. P. Benyon C., Iredale J. P.: Inhibition of apoptosis of activated hepatic stellate cells by tissue inhibitor of metalloproteinase- 1 is mediated via effects on matrix metalloproteinase inhibition. J. Biol. Chem. 2002, 277, 11069-11076.

40. Neer T. M.: A review of disorders of the gallbladder and extrahepatic biliary tract in the dog and cat. J. Vet. Intern. 1992, 6, 186-192.

41. Oishi Y., Tani K., Itamoto K., Haraguchi T., Taura Y.: Agenesis of hepatic lobes in a dog. Open Vet. J. 2018, 8, 485-488.

42. Owojuyigbe O. S., Firempong C. K., Larbie C., Komlaga G., Emikpe B. O. Hepatoprotective potential of Hura crepitans L.: A review of ethnomedical, phytochemical and pharmacological studies. J. Altern. Complement. Med. 2020, 1-10.

43. Ozer J.: The current state of serum biomarkers of hepatotoxicity. Toxicology 2008, 245, 194-205.

44. Pagan J. D., Crandell K.: Advances in Equine Nutrition II. Nottingham University Press, Nottingham 2001, 29-36.

45. Paulova J., Dvorak M., Kolouch F., Vanova L., Janeckova L.: Verification of the hepatoprotective and therapeutic effect of silymarin in experimental liver injury with tetrachloromethane in dogs. Vet. Med., Praha 1990, 35, 629-635.

46. Persiani S., Sala F., Cole R., Webber G., Caselli G., Vaghi P., Rovati L. C. Silibinin hemisuccinate binding to proteins in plasma and blood cell/plasma partitioning in mouse, rat, dog and man in vitro. Drug Metabol. Drug Interact. 2013, 28, 115-122.

47. Pietrangelo A., Montosi G., Garuti C., Contri M., Giovannini F., Ceccarelli D., Masini A.: Iron-induced oxidant stress in nonparenchymal liver cells: Mitochondrial derangement and fibrosis in acutely iron-dosed gerbils and its prevention by silybin. J. Bioenerg. Biomembr. 2002, 34, 67-79.

48. Polyak S. J., Morishima C.: Identification of hepatoprotective flavonolignans from silymarin. PNAS 2010, 107, 13, 5995-5999.

49. Povero D., Eguchi A., Li H., Johnson C. D., Papouchado B. G., Wree A., Messer K., Feldstein A. E.: Circulating extracellular vesicles with specific proteome and liver microRNAs are potential biomarkers for liver injury in experimental fatty liver disease. PLoS ONE 2014, 9, 12.

50. Rallis T., Day M. J., Saridomichelakis M. N., Adamama-Moraitou K. K., Papazoglou L., Fytianou A., Koutinas A.: Chronic hepatitis associated with canine leishmaniosis (Leishmania infantum): A clinicopathological study of 26 cases. J. Comp. Pathol. 2005, 132, 145-152.

51. Redman C. M., Xia H.: Fibrinogen biosynthesis: assembly, intracellular degradation, and association with lipid synthesis and secretion. Ann. N. Y. Acad. Sci. 2001, 480-495.

52. Rothuizen J., Bunch S., Charles J., Cullen J., Desmet V., Szatmari V., Twerdt D., Ingh T., Winkle T., Washabau R.: WSAVA Standards for Clinical 
and Histological Diagnosis of Canine and Feline Liver Diseases. Elsevier 2006

53. Santos D. I., Saraiva J. M. A., Vicente A. A., Moldão-Martins M.: Methods for determining bioavailability and bioaccessibility of bioactive compounds and nutrients. Innovative Thermal and Non-Thermal Processing, Bioaccessibility and Bioavailability of Nutrients and Bioactive Compounds 2019, 23-54

54. Serrano G., Charalambous M., Devriendt N., de Rooster H., Mortier F., Paepe D.: Treatment of congenital extrahepatic portosystemic shunts in dogs: A systematic review and meta-analysis. J. Vet. Intern. Med. 2019, 33, 1865 -1879 .

55. Sgorlon S., Stefanon B., Sandri M., Colitti M.: Nutrigenomic activity of plant derived compounds in health and disease: Results of a dietary intervention study in dog. Res. Vet. Sci. 2016, 109, 142-148.

56. Shaarawy S. M., Tohamy A. A., Elgendy S. M., Elmageed Z. Y., Bahnasy A.: Protective effects of garlic and silymarin on NDEA-induced rats hepatotoxicity. Int. J. Biol. Sci. 2009, 5, 549-557.

57. Shaymaa I. S., Abeer A. A. E., Faten F. M.: Cytoprotective effect of silymarin on cisplatin induced hepatotoxicity and bone marrow toxicity in rats. Asian J. Anim. Sci. 2017, 11, 140-152.

58. Skorupski K. A., Hammond G. M., Irish A. M., Kent M. S., Guerrero T. A., Rodriguez C. O., Griffin D. W.: Prospective randomized clinical trial assessing the efficacy of Denamarin for prevention of CCNU-induced hepatopathy in tumor-bearing dogs. J. Vet. Intern. Med. 2011, 25, 838-845.

59. Soleimani V., Delghandi P. S., Moallem S. A., Karimi G.: Safety and toxicity of silymarin, the major constituent of milk thistle extract: An updated review. Phytother. Res. 2019, 33, 1627-1638.

60. Sonnenbichler J., Zelt I.: Stimulating influence of a flavonolignane derivative on proliferation, RNA synthesis and protein synthesis in liver cells. Assessment and Management of Hepatobiliary Disease, Springer, Berlin 1987.
61. Steiner T:: Phytogenics in Animal Nutrition Natural Concepts to Optimize Gut Health and Performance. Notthingam University Press 2009.

62. Vandeweerd J.-M., Cambier C., Gustin P.: Nutraceuticals for canine liver disease. Vet. Clin. North Am. Small Anim. Pract. 2013, 43, 1171-1179.

63. Vereckei A., Besch H. R., Zipes D. P.: Combined amiodarone and silymarin treatment, but not amiodarone alone, prevents sustained atrial flutter in dogs. J. Cardiovasc. Electrophysiol. 2003, 14, 861-867.

64. Vogel G., Tuchweber B., Trost W., Mengs U.: Protection by silibinin against Amanita phalloides intoxication in beagles. Toxicol. Appl. Pharmacol. 1984, $73,355-362$.

65. Wellington K., Adis B. J.: Silymarin: A review of its clinical properties in the management of hepatic disorders. Bio Drugs 2001, 15, 465-489.

66. Wu J. W., Lin L. C., Tsai T. H.: Drug-drug interactions of silymarin on the perspective of pharmacokinetics. J. Ethnopharmacol. 2009, 121, 185-193.

67. Xie Y., Zhang D., Zhang J., Yuan J.: Metabolism, transport and drug-drug interactions of silymarin. Molecules 2019, 24, 3693.

68. Yadav N. P., Pal A., Shanker K., Bawankule D. U., Gupta A. K., Darokar M. P., Suman S. P.: Synergistic effect of silymarin and standardized extract of Phyllanthus amarus against CCl4-induced hepatotoxicity in Rattus norvegicus. Phytomedicine 2008, 15, 1053-1061.

69. Yamada T., Kakinoki M., Totsuka K., Ashida Y., Nishizono K., Tsuchiya R., Kobayashi K.: Purification of canine alpha-fetoprotein and alpha-fetoprotein values in dogs. Vet. Immunol. Immunopathol. 1995, 47, 25-33.

70. Yan-yu X., Yunmei S., Zhipeng C., Qineng P.: Preparation of silymarin proliposome: A new way to increase oral bioavailability of silymarin in beagle dogs. Int. J. Pharm. 2006, 319, 162-168.

Corresponding author: Maciej Gogulski, DVM, Szydlowska 43, 60-656 Poznań, Poland; email: maciej.gogulski@up.poznan.pl 